\title{
The Great Mimic Again? A Case of Tuberculosis Knee
}

\author{
Teo SH, MBBS (IMU), Teh KK, MS Orth (UM), L Azura, MS Orth (UM), Ng YO, FRCS (Ed) \\ Orthopaedic, Ampang Hospital, Kuala Lumpur, Malaysia
}

\begin{abstract}
Tuberculosis (TB), once a disease confined to undeveloped or developing nations is currently in resurgence due to pandemic human immunodeficiency virus infection and immigration from endemic areas. TB is also known as the 'great mimicker'. Extra-pulmonary tuberculosis affecting the knee is rare in all forms of TB (0.1-0.3\%). Here, we report a case of isolated highly erosive TB knee in a previously fit Burmese migrant worker. He presented with after a history of fall into a drain. The patient also reported pain and swelling over his left knee for the previous three years. He had been treated for a bacterial infection of the knee in another hospital but defaulted due to financial constraints. Arthrotomy of the knee was performed including washout. Diagnosis of TB of the knee was made based on the synovial fluid and tissue culture. Treatment with antituberculosis drugs was then initiated.
\end{abstract}

Key Words:

Tuberculosis Of The Knee, Extrapulmonary Tuberculosis

\section{INTRODUCTION}

Extra-pulmonary tuberculosis (EPTB) is observed in approximately $20 \%$ of all tuberculosis (TB) cases, but incidence has increased in recent years ${ }^{1}$. Osteoarticular TB comprises approximately $10 \%$ of EPTB cases, but this number is also on the rise1. Of note, mono-articular TB of the knee is rare in all form of EPTB. As in other countries, the re-emergence of TB is attributed to high influx of foreign workers, and increasing prevalence of immunosuppressed HIV/AIDS patients ${ }^{2}$. Here, we report a case of TB of the knee in which the diagnosis was delayed; we highlight this uncommon but increasingly occurring presentation, which may initially present as an uncomplicated septic arthritis.

\section{CASE REPORT}

A 35- year-old Myanmar migrant male worker was referred to us from a local clinic for a painful and swollen left knee associated with pus discharge for three months.

He had history of fall into a drain three years prior to presentation, which may or may not be related to the presenting complaint; however he started to have pain and swelling over his left knee after that incident. For the initial three years, he only sought treatment at local clinic and was prescribed analgesics. Three months prior to presentation to our hospital, he developed purulent discharge from the knee with progressive worsening of pain, swelling and inability to bear weight. He first sought treatment at another hospital, was admitted and treated for bacterial septic arthritis with intravenous antibiotics. He was scheduled for a knee washout but defaulted due to financial reasons. The International Organization of Migration then referred him to our clinic. The patient reported no recent respiratory infection, night sweat and loss of appetite. However, he claimed that there was significant weight loss, and denied use of intravenous drugs.

Physical examination revealed a swollen left knee with fixed flexion deformity of 10 degree with a sinus tract and pus discharge over the medial aspect. Examination of other joints was normal, and the rest of the systemic examination was unremarkable. Laboratory examination revealed a haemoglobin level of $10.6 \mathrm{~g} / \mathrm{dl}$, total leukocyte count of 7,900/uL (neutrophils, 72\%; lymphocytes, 17\%; monocytes, $6 \%$; eosinophils, 4\%; basophils, 1\%), and erythrocyte sedimentation rate of $75 \mathrm{~mm} / \mathrm{hr}$. Human immunodeficiency virus (HIV), Hepatitis $\mathrm{B}$ and $\mathrm{C}$ virus screening was non reactive. Left knee radiograph revealed several eroded areas over the femoral-tibial joint and narrowing of the joint space (Figure 1). The patient was admitted with a working diagnosis of TB of the knee. Differential diagnosis included septic arthritis, TB of the knee, inflammatory arthritis or malignancy.

A chest radiograph revealed an ill-defined opacity in the left upper lobe. Test for purified protein derivative (PPD) was not reactive. While we completed a TB workup, an urgent knee washout and biopsy was scheduled as the knee appeared to be infected. We attempted to washout the knee arthroscopically but the procedure was abandoned due to the reduced joint space. Intraoperatively, the knee had thickened synovium with multiple pockets of necrotic tissue, cheesy material and pus surrounding the distal femur. Articular surfaces of the patella-femoral and tibiofemoral joint were destroyed. Specimen of tissue, pus and synovial fluid were sent for culture; sensitivities, acid fast bacilli and histopathologic examination (HPE).

Mycobacterium tuberculosis was cultured from synovial fluid. HPE of the tissue showed necrotising granulomatous inflammation, highly suggestive of mycobacterial infection 


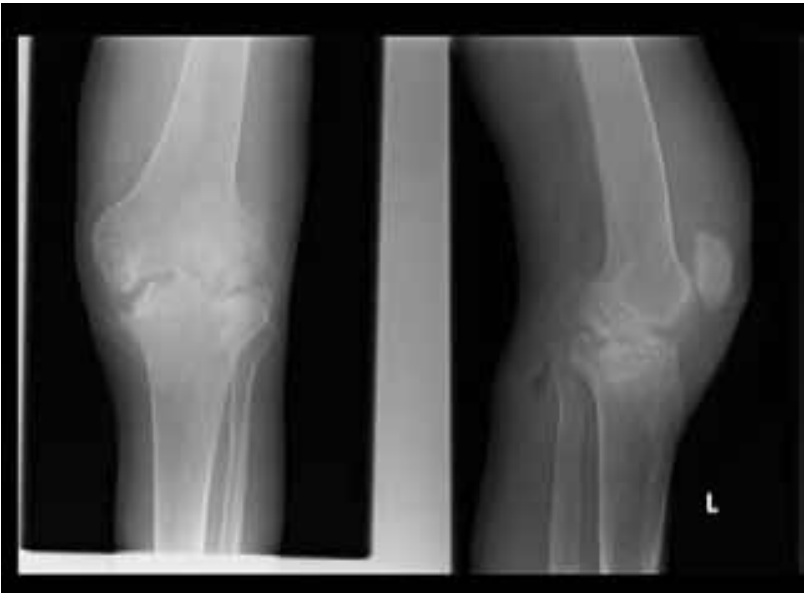

Fig. 1: Left knee X-Ray - revealed several erosive areas over the femoral-tibia joint and narrowing of the joint space.

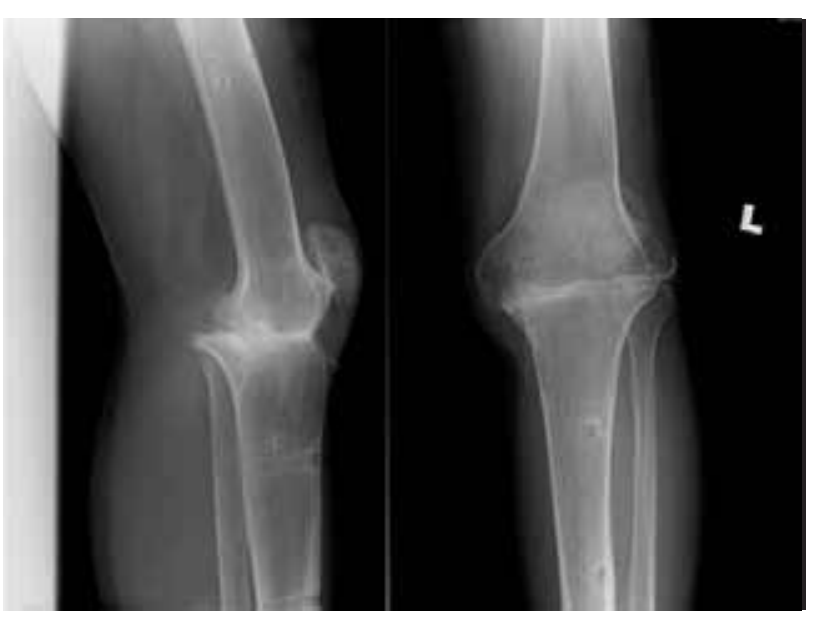

Fig. 3: Radiological union of the knee joint after 10 months.

Anti-TB drugs were prescribed and the patient completed 1 year of pharmacotherapy at the following doses: Isoniazid 300mg OD, Rifampicin 600mg OD, Pyrazinamide 1500mg OD, Ethambutol 1000mg OD, and Pyrazinamide 10mg OD. He subsequently underwent multiple debridements and was put on a full-length back slab for immobilisation.

His knee continued to have discharge, and was told to undergo arthrodesis; he initially refused such treatment. Five months later, the patient reconsidered and arthrodesis was performed using an Illizarov frame.

After fusion, discharge from the knee resolved and the patient returned to full weight bearing. Radiological union (Figure) was seen after 10 months and the frame was removed at that time. He was last reviewed at 11 months post-op; examination revealed complete resolution of the infection and the patient was walking with a short limb gait that improved with a heel raising orthotic.

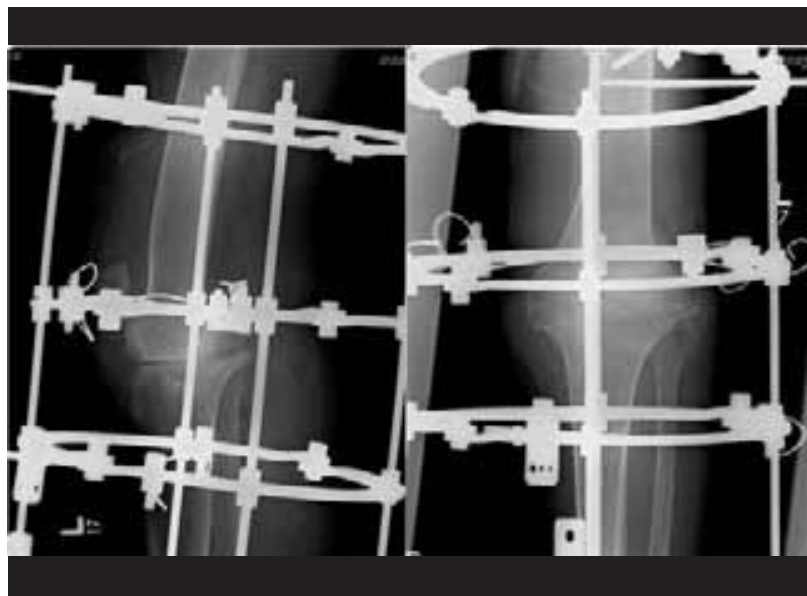

Fig. 2: Arthrodesis of the knee with illizarov frame.

\section{DISCUSSION}

Increasing incidence of osteoarticular TB has recently been reported. This type of TB is typically the result of a direct haematogenous spread of TB bacilli from a primary focus (e.g., pulmonary $(30 \%)$, genitourinary $(20 \%)$, or unidentified $(50 \%))$ site. Weight bearing joints are frequently involved in osteoarticular TB due to the effect of trauma ${ }^{1}$. The rising rate of TB is explained partly by the increased numbers of HIVinfected patients, intravenous drug abusers and the influx of foreign workers, as was the case in the patient described here.

Diagnosis of tuberculosis arthritis is often overlooked especially during early presentation of the disease. In this case, the patient was initially treated with analgesia and later for bacteria septic arthritis. These errors in treatment occur because of the subtle nature of the symptoms; full diagnostic evaluations are often not undertaken until the disease has progressed ${ }^{1}$.

Tuberculosis of bones and joints are usually diagnosed following radiologic examination and tissue biopsy. The identification of $M$. tuberculosis is essential for the diagnosis of tuberculous arthritis. This can be based on either HPE or culture. Due to the frequency of associated tuberculosis in the lungs and kidneys, culture of sputum and urine specimens can be helpful ${ }^{3}$. Of note, however, acid-fast stain of joint fluid is positive in only $0-5 \%$ of those examined, and M. tuberculosis is isolated in approximately $60-89 \%$ of cases ${ }^{1,4}$. We were fortunate to obtain a positive culture in this case.

This case highlights the uncommon but increasingly recognized presentation of tuberculous arthritis of a joint. Such cases may initially present as uncomplicated septic arthritis. With the increasing prevalence of TB in our region, it is important to increase clinical awareness to ensure early detection and avoid diagnostic delay ${ }^{5}$. Finally, as illustrated in this case, certain people are unable to effectively gain access to health care services. Had it not been for the existence of special outreach programs to help marginalized people, this patient's diagnosis would have been further delayed. 


\section{REFERENCES}

1. Hopewell PC. Overview of Clinical Tuberculosis. In: Bloom BR, editor. Tuberculosis. Washington, D.C. American Society for Microbiology; 2005. P. 25-46.

2. Brennan PJ. Tuberculosis in the context of emerging and reemerging diseases, FEMS Immunol Med Microbiol. 1997; 18 (4): 2639.

3. Watts HG, Lifeso RM. Tuberculosis of Bones and joints. J Bone Joint Surg Am. 1996; 78(2): 288-98.

4. Iagnocco A, Coari G, Buzzi G, Guerrisi R, Valesini G. Magnetic resonance imaging of peripheral osteoarticular tuberculosis compared with Sonography and standard radiographs. Rheumatol Int. 2003; 23(4):195-7.

5. Chen YC, Hsu SW. Tuberculous arthritis mimic arthritis of Sjögren's syndrome: findings from sonography, computed tomography and magnetic resonance images. Eur J Radiol. 2001; 40(3): 232-5. 